\title{
Power-law scaling in dimension-to-biomass relationship of fish schools
}

\author{
Hiro-Sato Niwa $^{1}$ \\ Behavioral Ecology Section, National Research Institute of Fisheries Engineering, \\ Hasaki, Ibaraki 314-0421, Japan
}

\begin{abstract}
Motivated by the finding that there is some biological universality in the relationship between school geometry and school biomass of various pelagic fishes in various conditions, I here establish a scaling law for school dimensions: the school diameter increases as a power-law function of school biomass. The power-law exponent is extracted through the data collapse, and is close to $3 / 5$. This value of the exponent implies that the mean packing density decreases as the school biomass increases, and the packing structure displays a mass-fractal dimension of 5/3. By exploiting an analogy between school geometry and polymer chain statistics, I examine the behavioral algorithm governing the swollen conformation of large-sized schools of pelagics, and I explain the value of the exponent.
\end{abstract}

Key words: power-law scaling, data collapse, pelagic fish, school size, geometry

\section{Introduction}

\subsection{Brief history of studies on the packing geometry of fish schools}

Animal packing in social aggregations is of fundamental interest in ecology, and their conformations have been extensively studied (Parr, 1927; Svmons, 1971; Okubo and Chiang, 1974; Graves, 1976; Pitcher and Partridge, 1979; Aoki, 1980; Partridge, 1980, 1982; Dill et al., 1981; Aoki and Inagaki, 1988; Misund, 1993a; Parrish and Hamner (Eds.), 1997). Pioneer tank observations of pelagic

fishes (herring, sprat and mackerel) were conducted by Parr (1927), and the

1 Tel.: +81-479-44-5953; fax: +81-479-44-1875.

E-mail address: Hiro.S.Niwa@fra.affrc.go.jp (H.-S. Niwa).

Preprint submitted to Elsevier Science

17 February 2019 
"persistently uniform density" of a school was noted. From experiments on schools of saithe Pollachius virens, herring Clupea harengus and cod Gadus morhua cruising in a ten meters circular gantry tank, Pitcher and Partridge (1979) validated that all the fish in a school occupy a volume proportional to $N b^{3}$, where $N$ is the number of fish and $b$ is the mean nearest-neighbor distance (approximate to the mean fish-body length) in the school. Misund (1993a) reported from field observations that the number densities of herring schools are almost independent of the dimensional size of school but are an order of magnitude lower than the packing densities observed when schooling in small tanks.

Tauti and colleagues (Tauti and Mivosi, 1929; Tauti and Hudino, 1929; Tauti and Yasuda, 1929, 1930, 1933a,b) had experimentally shown that a fish school can be viewed as a group of inorganic particles and treated with the methods of physics. Breden (1954) explicitly applied physical equations to such fish schools. Recently, a number of theoretical and numerical models of schooling and flocking behavior have been studied by biologists, mathematicians and physicists (Okubo and Levin, 2001; Camazine et al., 2001; Vicsek (Ed.), 2001). As for the animal group geometry, many models for social aggregations, however, predict that densities increase as the group size (in number) increases [overviews of such models are given in Refs. (Warburton and Lazarus, 1991; Beecham and Farnsworth, 1999)]. Mogilner et al. (2003) mathematically tackled this problem of constant density and revealed the condition for a well-spaced group, i.e. what class of mutual interactions result in a relatively constant individual distance in the interior of the aggregate.

More recently, by means of underwater acoustics, the school sizes (in number or biomass of fish) have been quantitatively measured for different values of the dimensional size of schools in the wild. Precise data on conformations of large-sized schools of pelegic fishes became available rapidly, which were extremely helpful in elucidating a certain geometric law, i.e. the relation between dimensional and biomass sizes of pelagic fish schools, bringing about some important changes in our viewpoints. Misund and colleagues (Misund, 1990; Misund et al., 1992; Misund, 1993b; Misund et al., 1995, 1996; Coetzee, 2000; Misund and Coetzee, 2000; Misund et al., 2003) found that the powerlaw scaling in dimension-to-biomass relationship exists, and is robust across a broad range of pelagic species as well as across diverse environments. They demonstrated that if the biomass $N$ in a school is, say, doubled, the crosssectional area of a school is increased by a factor $2^{2 \nu}$, i.e.

$$
\text { cross-sectional area } \propto(\text { biomass })^{2 \nu},
$$

and that the exponent $\nu$ looks universal, reading 0.5 (from the field data $\nu$ ranges from 0.415 to 0.77 ). The geometric law they found implies that the mean density of a school scales as $N^{1-3 \nu}$ in three dimensions of space and the 
conformation of social aggregations swells (i.e. $\nu>1 / 3$ ). Such a relationship as Eq.(1) with $\nu=0.5$ has been utilized for the dimensions to biomass conversion (e.g. transforming the school diameter $R$ to the number $N$ of fish) in analyzing school-size distributions (Anderson, 1981; Niwa, 1996b, 1998, 2003, 2004a).

It might come as a surprise that packing densities decrease as the group size increases, contrary to previous observations and predictions. Laboratory observations for school geometry and internal structure have been made exclusively in small tanks, which generally show the constant density of fish that the school volume is proportional to the number of individuals. In all set-ups, the factors resulting in homogeneous, cohesive school may be especially pronounced, and it therefore seems that the quantified structure is skewed. Pitcher and Parrish (1993) claimed that homogeneity in schools has been over-emphasized. In-situ observations of herring and sprat schools with a high resolution sonar revealed that the packing structure within the schools is rather heterogeneous (Cushing, 1977). This has been confirmed by measurements of free-swimming schools using photography and high-resolution echo integration, which showed that the packing density distribution in capelin Mallotus villosus and clupeoid schools varies considerably (Fréon et al., 1992; Misund, 1993a). Regions of high density are usually found within the schools, and even empty vacuoles have been recorded. Misund and Floen (1993) observed by repeated echo integration that there were large variations in internal packing density of herring schools (i.e. high-density regions or empty lacunas within a school), and that the packing density structure was quasi-stationary. Besides artificial environments in small tanks, the discrepancy in former observations could have been caused by too small numbers of fish in the schools. For instance, in Pitcher and Partridge (1979), $N$ takes a few tens of fish. Since such a geometric law above is always defined only in a certain limit (de Gennes, 1979), the scaling in the dimensionto-biomass relationship is expected to hold for large-sized schools of pelagics.

In this paper, the exponent $\nu$ is estimated according to the established universal scaling law in the school-size distribution of pelagic fishes (Niwa, 2003, 2004a): choose the suitable value of $\nu$ to achieve the best data-collapse on the size distributions in terms of the school dimension. Notice that the dimensionto-biomass relationship is a property of the single school and the scaling exponent $\nu$ is determined by the behavioral algorithm of fish schooling at individual level, while the scaling in the school-size distribution emerges from the interschool interactions at population level (i.e. a global property of the interacting school system). 


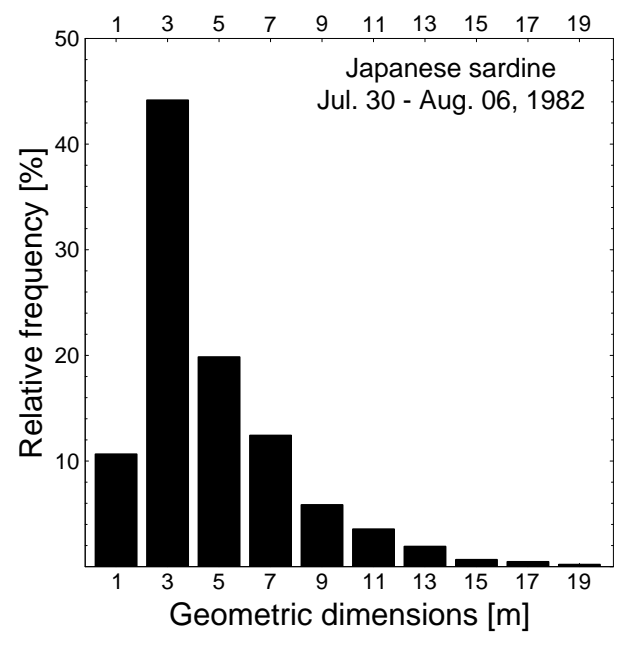

Fig. 1. Vertical dimension (in meters) of Japanese sardine schools. Data from the acoustic survey by Hara (1990) off southeastern Hokkaido in the summer, 1982. The same data are available in Hara (1984). Small schools of vertical dimensions in the first bin ( $<$ two meters) may be stray ones.

\subsection{Scaling in school-size distributions}

Animal group size is a focal issue in ecology that, in contrast to scaling, has introduced a single preferred size (i.e. optimal or compromise size) for any organism living in groups (Pulliam and Caraco, 1984; Higashi and Yamamura, 1993; Niwa, 1996b; Hoare et al., 2004). Figure 1 shows an example of the histogram of school dimensions of Japanese sardine Sardinops melanostictus. A peak frequency and a right skew are typical of pelagic fishes (Anderson, 1981; Niwa, 1996b, 1998). Their linear dimension, e.g. the vertical thickness, of the school falls into a certain range below a few tens of meters. One fish may not be the right atomic unit in schooling, since field observations suggest that no school exists under a certain minimal size. This may cause binned data of school sizes to exhibit the fake peak frequency. Anderson (1981) and Niwa (1996b) have ignored slowly decaying (or fat-tailed) distributions, including scaling laws, in such data of school sizes short ranged with a fake peak frequency (the possibility of power laws was already presented in their models but not exploited). A traditional, widely used Gauss statistics says that, for the data from Hara (1990) shown in Fig.1, finding sardine schools ranging from 18 to 20 meters in vertical thickness should only occur about once every $10^{9}$ detections of schools [for detail, consult Niwa (2004a)]. In other words, it is not the real world! Aquatic observations actually say that finding such schools occurs about once every 500 detections. The probability that such schools are found is $10^{6}$ times as large!

The possibility of scaling in such distributions was found by Bonabeau and Dagorn (1995). Fat-tailed distributions have been found to quite generally character- 
ize the size heterogeneity of pelagic fish schools (Niwa, 1998; Bonabeau et al., 1998, 1999). Only lately, Niwa (2003, 2004a) showed that the school-size distribution of pelagic fishes has the universal functional form of a power-law decay and a crossover towards the exponential distribution.

\subsection{Scaling and data collapse in ecology}

Scaling laws have been found to characterize many patterns in ecological systems (Azovskv, 2000; Chave and Levin, 2003; Hallev et al., 2004). There is an empirical rule about the relationship between the areas $(L)$ of island and the numbers $(S)$ of species on them: whether counting birds, butterflies, plants or copepods in ponds, a tenfold smaller area contains roughly half the species. This can be fitted by a power-law function

$$
S(L) \propto L^{z}
$$

with the exponent $z \approx 0.25$ (Preston, 1962; MacArthur and Wilson, 1967; Harte et al., 1999; Mav, 1999). The species-area relationship is widely used in rough assessments of likely future rates of species extinction (because observed rates of tropical deforestation are equated to loss of habitat area, we can assess the annual production of species committed to extinction). So, a more secure understanding of such relationships has great practical importance (Mav), 1999). Banavar et al. (1999) applied finite-size scaling (Fisher and Barber, 1972; Binder and Heermann, 1988) to test the hypothesis of scaling invariance in the species-area relationship resting on a model proposed by Harte et al. (1999) for the species abundance distribution. Following the conventional notation in the physics literature, the system sizes are hereafter denoted by $L$.

The finite-size scaling (FSS) hypothesis assumes that the fraction of objects with size $n$ for a finite system of size $L$ is written, with a scaling exponent $A$, as

$$
P(n ; L)=L^{-A} F\left(n / L^{A}\right),
$$

where $F$ is a universal scaling function. $P(n ; L)$ reads, for instance, the speciesabundance distribution, which defines the probability that any given species on a census patch of area $L$ has $n$ individuals, where $P(n ; L) S(L)$ is the expected number of species with $n$ individuals. $L^{A}$ is a measure of the width of the probability distribution $P(n ; L)$, e.g. the mean or the standard deviation of the distribution. Equation (3) expresses the principle that the behavior of the finite system, i.e. $P(n ; L)$, is controlled by the ratio $n / L^{A}$. We test the FSS hypothesis by data collapsing: when $y=L^{A} P(n ; L)$ is plotted versus $x=n / L^{A}$, if the distributions across different $L$ 's fall on a single curve (e.g. collapsing distinct abundance distributions measured in different areas and with different total numbers of individuals onto one scaling curve), then we 
should be able to identify a scaling function $F(x)$. In order to determine the exponent $A$, we try a best-fitting procedure such that the family of curves $P(n ; L)$ collapses onto a single curve as well as possible. We then obtain other scaling exponents (e.g. $z$ of the species-area relationship) resting on the scaling relation between scaling exponents (Goldenfeld, 1992). By noting that the total number of individuals of all species is equal to $\sum_{n} n P(n ; L) S(L)$, the species-area relationship leads to a scaling relation

$$
A+z=1
$$

assuming that the total number of individuals of all species is proportional to area $L$ (Banavar et al., 1999).

The universal scaling is fundamental to data collapse. The power-law scaling emerges as ubiquitous properties in ecology. In statistical analysis in ecology, data collapsing across different environments or species has been, however, observed only lately in dynamics of breeding bird populations (Keitt and Stanlev, 1998; Keitt et al., 2002), food web structure (Camacho et al.,,2002), microbial body-mass spectra in marine ecosystems (Rinaldo et al., 2002), school-size distributions of pelagic fishes (Niwa, 2003, 2004a), and in the context of ecological economics, capture fisheries productions of countries (Niwa, 2004b).

\section{Scaling in dimension-to-biomass relationship}

\subsection{Estimation of the exponent $\nu$ through data collapse}

In order to understand the geometric properties of school configurations, the school biomass $N$ must be measured for different values of dimensional size $R$, and we must compare them. Misund and colleagues extensively performed the simultaneous observations of the values of $R$ and $N$ for pelagics by the following two methods: (i) two-dimensional (cross-sectional) acoustic measurements (unit in square meters) and subsequent purse seine capture (unit in tonnes) of schools (Misund, 1990, 1993b), and (ii) acoustic measurements of the threedimensional structures and backscattered echo energy (Misund et al., 1992, 1995, 1996; Coetzee, 2000; Misund and Coetzee, 2000; Misund et al., 2003). The surveys were conducted on stocks of anchovy Engraulis capensis, herring Clupea harengus, horse mackerel Trachurus trachurus capensis, mackerel Scomber scombrus, pilchard Sardinops ocellatus, round herring Etrumeus whiteheadi, saithe Pollachius virens, sardine Sardinops sagax, and sprat Sprattus sprattus, in different seasons and geographic regions (the Barents Sea, the North Sea, the Norwegian Sea, the northeastern Atlantic and off Namibia, and off the coast of South Africa). They found that there is some biological 
universality in the dimensions-to-biomass relationships, Eq.(1). Their finding implies that the radius $R$ of the school scales as

$$
R=(\text { constant }) \times N^{\nu}
$$

in a statistical sense. $N$, denoting the school biomass, is hereafter defined by the number of fish in a school.

Now I propose another way to establish the scaling law for school dimensions, Eq. (5), by applying universal scaling law in fish school-biomass distributions (Niwa, 2003, 2004a). The biomass distributions $W(N)$ follow a power law with exponent $\beta=1$ up to a cut-off size $\langle N\rangle_{P}$,

$$
W(N)=N^{-\beta} P\left(N /\langle N\rangle_{P}\right)
$$

where $P(x)$ is a crossover scaling function with a strong drop for $x>1$, and the cut-off size (crossover size from power-law to exponential decay) is calculated from the biomass histogram data $\left\{\left(N_{i}, W_{i}\right) \mid i=1,2, \ldots\right\}$,

$$
\langle N\rangle_{P}=\frac{\sum_{i} N_{i}^{2} W_{i} \Delta N}{\sum_{i} N_{i} W_{i} \Delta N}
$$

where histogram bins are chosen with width $\Delta N$.

The dimension data of fish schools are binned with width $\Delta R$, giving the set of frequencies $\left\{\left(R_{i}, W_{i}^{(\mathrm{G})}\right) \mid i=1,2, \ldots\right\}$. From Eqs.(5) and (6), the distribution of geometric dimensions of fish schools is represented as

$$
W^{(\mathrm{G})}(R)=R^{-1} P^{(\mathrm{G})}\left(R /\langle R\rangle_{P}\right)
$$

where

$$
\langle R\rangle_{P}=\langle N\rangle_{P}^{\nu}
$$

and

$$
P^{(\mathrm{G})}(x)=P\left(x^{1 / \nu}\right) .
$$

Therefore, the school-dimension distribution follows a power law with the same exponent " -1 " as the school-biomass distribution. The power-law distribution $W^{(\mathrm{G})}(R)$ is truncated at a cut-off size $\langle R\rangle_{P}$, which is also calculated from histogram data of school geometric dimensions,

$$
\langle R\rangle_{P}=\left[\frac{\sum_{i} R_{i}^{2 / \nu} W_{i}^{(\mathrm{G})} \Delta R}{\sum_{i} R_{i}^{1 / \nu} W_{i}^{(\mathrm{G})} \Delta R}\right]^{\nu} .
$$

The following normalizations are adopted for the scaling function $P^{(\mathrm{G})}(x)$ and the histogram data of geometric dimensions of fish schools, because the cutoff size $\langle N\rangle_{P}$ is proportional to the total number of fish in the school system 
Niwa (2003, 2004a); see also Eq.(23)]:

$$
\int_{0}^{\infty} x^{1 / \nu-1} P^{(\mathrm{G})}(x) \mathrm{d} x=1,
$$

and

$$
\sum_{i} R_{i}^{1 / \nu} W_{i}^{(\mathrm{G})} \Delta R=\langle R\rangle_{P}^{1 / \nu},
$$

respectively.

Fat-tailed school-size distributions are necessarily truncated because the population is finite. Since the size $\langle R\rangle_{P}$ depends on the exponent $\nu$ following Eq.(11), so that we can determine the value of $\nu$ by evaluating the location of the cut-off in the power-law distribution $W^{(\mathrm{G})}(R)$. The ordinary least squares regressions might, however, lead to a "wrong" estimation of the exponent (Niwa, 1998; Bonabeau et al., 1998, 1999). I make use of the data collapse to extract the "right" exponent. From Eqs.(8) and (12), when $y=W^{(\mathrm{G})}\langle R\rangle_{P}$ is plotted against $x=R /\langle R\rangle_{P}$ with correct parameter $\nu$, all the empirical data should collapse onto each other. The power-law exponent of school-dimension distributions, $\nu$, is then evaluated through data collapse. Let us search for the value of $\nu$ that places all the points most accurately on a single curve. We use a set of histogram data of vertical dimension of Japanese sardine Sardinops melanostictus schools, from 22 acoustic surveys by Hara (1990) off southeastern Hokkaido for seven years, 1981-1987. Hara (1986) reported that Japanese sardine migrate as a huge-sized school in number from a few hundreds of thousands to a few million of fish. To obtain the best data collapse, the $x$-axis is divided into bins (Fig.2a), and for each bin two-dimensional variance

$$
\epsilon=\left(\sigma_{x} / \mu_{x}\right)^{2}+\left(\sigma_{y} / \mu_{y}\right)^{2}
$$

is calculated, where $\sigma_{x}$ and $\sigma_{y}$ denote the standard deviation of the mean $\mu_{x}$ and $\mu_{y}$, respectively. The parameter $\nu$ is then estimated at value that minimize the mean of two-dimensional variance for the bins (Fig.2b). The mean of twodimensional variance, $\bar{\epsilon}$, is a measure to determine the goodness of collapse (Lillo et al., 2002, 2003; Niwa, 2004a). A good data collapse can be obtained by using the value $\nu \approx 0.6$. The resulting plot of empirical school data is shown in Fig.2a. Experimentally fitting the parameter $\nu$ to achieve a good data collapse, " $3 / 5$ ", is reminiscent of the Flory value of the exponent in a powerlaw dependence of the coil radius of a polymer chain (in three-dimensional solutions) on the degree of polymerization (Florv, 1953; de Gennes, 1979).

Here we see that the power-law regime of the distribution is too short, which misled Anderson (1981) and Niwa (1996b) into overlooking the power-law distributions of school sizes. Notice that many power laws that appeared in the ecology literature span less than two orders of magnitude of scale (Hallev et al., 2004). The power law range of too few scales is not unique 

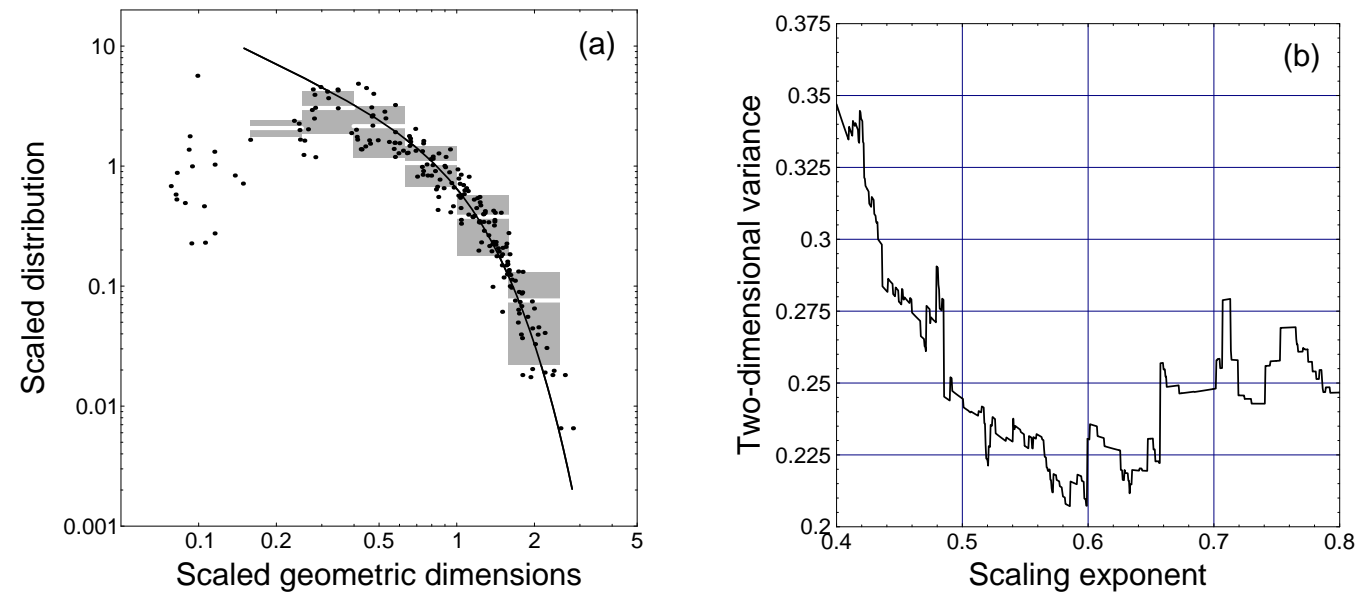

Fig. 2. Data collapse to extract the exponent $\nu$. (a) Scaled distribution of geometric dimensions of sardine schools. $y=W^{(\mathrm{G})}\langle R\rangle_{P}$ is plotted versus $x=R /\langle R\rangle_{P}$ with $\nu=0.6$ on double-logarithmic scale. The bins are chosen equally spaced on a logarithmic scale as $x \in\left[10^{-1+k / 5}, 10^{-1+(k+1) / 5}\right)$ with $k=1,2, \ldots, 6$. The rectangle in gray reads the interval $\mu_{y} \pm \sigma_{y}$, i.e. the error $\sigma_{y}$ on the mean $\mu_{y}$ (indicated by the slit) for each bin. The solid line is a prediction of the mean-field theory (Niwa, 2003). (b) The mean of two-dimensional variance, $\bar{\epsilon}$, versus the power-law exponent $\nu$. Although $\bar{\epsilon}$ shows noisy fluctuations, it takes a minimum around $\nu=0.6$. Data from Hara (1990) are analyzed.

to ecology; the largest numbers of power laws reported in the physical science are for small ranges (Hamburger et al., 1996).

\subsection{Retest of the FSS in school-biomass distributions}

The acoustic-survey data are converted into a school-biomass histogram as follows

$$
W(N) \Delta N \propto W^{(\mathrm{G})}(R) R^{1 / \nu-1} \Delta R .
$$

We now crosscheck the value of $\nu$ through finite-size scaling (FSS) analysis of school-biomass distribution (Niwa, 2004a). Since the finite population size causes the truncation of power-law distribution $W(N) \propto N^{-\beta}$, there is a welldefined quantity

$$
L=\frac{\sum_{i} N_{i}^{1+\beta} W_{i} \Delta N}{\sum_{i} N_{i}^{\beta} W_{i} \Delta N},
$$

which depends on the system population size. In order to characterize the finite size effects, FSS hypothesis is used: the distribution function depends on $N$ only through the ratio $N / L^{A}$,

$$
W(N ; L) \mathrm{d} N=L^{-B} F\left(N / L^{A}\right) \mathrm{d}\left(N / L^{A}\right),
$$


where $F(x)$ is a universal function independent of fish population size. The prefactor $L^{-B}$ is required to ensure the normalization

$$
\sum_{i} N_{i}^{\beta} W_{i} \Delta N=1
$$

where $P(N)$ [三 $\left.N^{\beta} W(N)\right]$ now represents the probability distribution of school-biomass sizes. From the FSS hypothesis, it is expected that when $W L^{A+B}$ is plotted against $N / L^{A}$ with correct parameters $A$ and $B$ all the data collapse onto a single curve. The power-law exponent of biomass distributions, $\beta$, is then evaluated through FSS analysis. The value of $B / A$ is the estimate of the power-law exponent

$$
\beta=\frac{B}{A}
$$

Let us analyze a set of 22 acoustic-survey data of sardine schools (same as Fig.2) converted into biomass histograms by using Eq.(15) with $\nu=3 / 5$. In a simultaneous best-fitting procedure (Fig.3), a good data collapse can be obtained by using the values $A \approx 1$ and $B \approx 1$. The power-law exponent derived from the FSS collapse is $\beta \approx 1$. The resulting plot is shown in Fig.3a. The school-biomass distribution follows a power-law decay with exponent -1 , and is truncated at the cut-off size of Eq.(7). The FSS collapse confirms the scaling laws for school sizes, Eq.(5) with $\nu=3 / 5$ and Eq.(6) with $\beta=1$.

Finite-size scaling techniques have been applied to ecology and shown to predict scaling relations between scaling exponents in relative abundance of species [Eq.(4)] (Banavar et al., 1999; Aii and Goldenfeld, 2001), in dynamics of breeding bird populations (Keitt et al., 2002), in biomass-size distributions of seston (Rinaldo et al., 2002), and in exploitation of fish stocks (Niwa, 2004b). In school-size statistics, we expect to have a scaling relation. We now choose the normalization

$$
\sum_{i} N_{i} W_{i} \Delta N=\Phi
$$

where $\Phi$ denotes the total fish population in the school system $\left(\sum_{i} W_{i} \Delta N\right.$ gives the total number of schools). Since Eq.(16) implies $L^{A} \propto \Phi^{\gamma}$ with a scaling exponent $\gamma$, the FSS relation for the school-biomass distribution is written as

$$
W(N)=N^{-\beta} P\left(N / \Phi^{\gamma}\right) .
$$

Accordingly, the normalization of Eq.(20) yields the scaling relation

$$
2-\beta=1 / \gamma
$$

The best-fitting value in data collapse, $\beta \approx 1$, gives $\gamma \approx 1$. As a consequence, the location of the cut-off in the power-law distribution of school sizes simply 

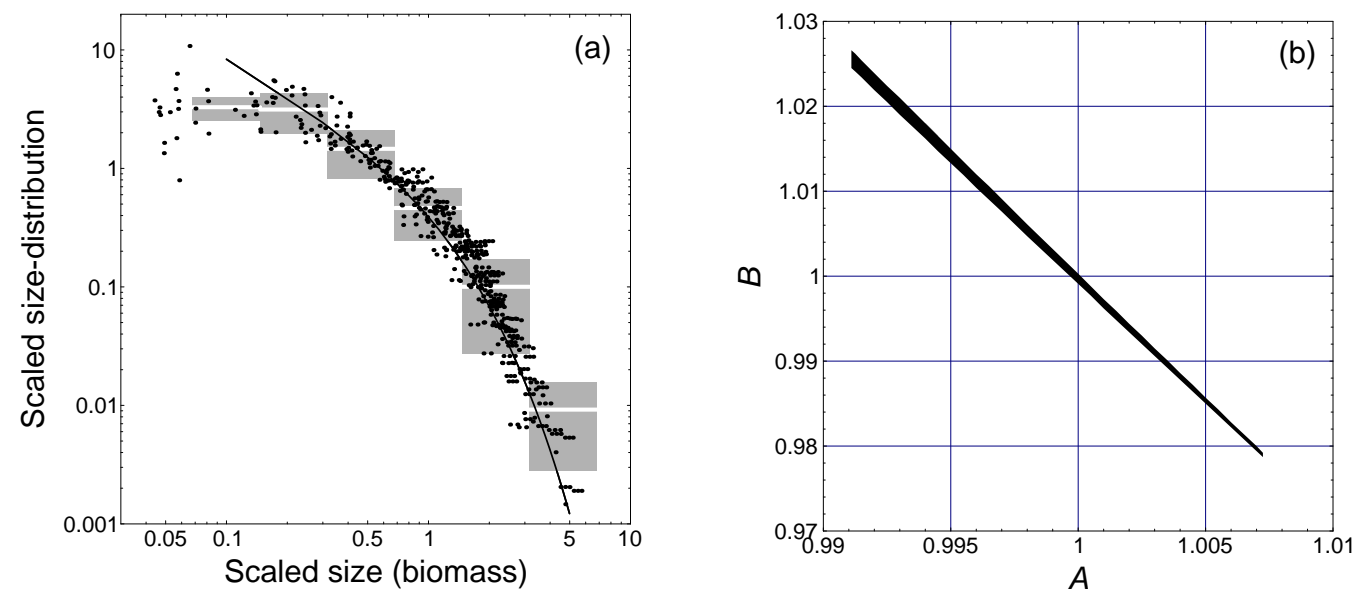

Fig. 3. FSS analysis of school-size data. (a) FSS plot of the biomass distribution on double-logarithmic scale. Dimension data of sardine schools (same as Fig.2) are converted by Eq.(15) with $\nu=3 / 5$. Here $y=W L^{A+B}$ is plotted versus $x=N / L^{A}$ with $A=B=1$. Two-dimensional variances [same as Eq.(14)] are calculated for bins chosen equally spaced on a logarithmic scale as $x \in\left[10^{-1+(k-0.5) / 3}, 10^{-1+(k+0.5) / 3}\right)$ with $k=0,1, \ldots, 5$. The rectangle in gray is same as Fig.2. The solid line is a prediction of the mean-field theory (Niwa, 2003). (b) The region of the $A B$-plane in which the minimum of the mean of two-dimensional variance exists. A measure of data collapse for scaling, i.e. the mean of two-dimensional variance, $\bar{\epsilon}$, takes a minimum $\bar{\epsilon}_{\text {min }}$ for the right choice of $(A, B)$. The minimum is found with the precision i.e. width of the minimum, $\Delta \epsilon=10^{-3}$ in black region $\left(\Delta \epsilon / \bar{\epsilon}_{\min } \approx 3.12 \times 10^{-3}\right)$. The values of the parameters lie in the intervals $A=0.999 \pm 0.008$ and $B=1.003 \pm 0.024$, and therefore $\beta=1.004 \pm 0.032$.

reads

$$
\langle N\rangle_{P} \propto \Phi
$$

which is verified by means of extensive numerical simulations (Niwa, 2003, 2004a).

\section{Behavioral algorithm of fish schooling}

\subsection{Gaussian model}

Let us now investigate cohesive motion of schools in a three-dimensional space from the viewpoint of the behavioral algorithms which govern their formation and dynamics: attraction between neighbors maintains cohesion of the school; a tendency to align with neighbors produces collective motion of the school. The minimal model of cohesion is a linkage of neighbors consisting of harmonic spring, because the inter-fish distance follows a Gaussian distribution 
(Dill et al., 1981; Partridge, 1982; Niwa, 1996a). Using the relative coordinates to the center of school, the equation of motion of fish (as noisy self-propelled particles) in a large school of size $N$ is written by a one-body approximation as the following Langevin equation (Niwa, 1994, 1996a):

$$
\frac{\mathrm{d}^{2} \boldsymbol{r}}{\mathrm{d} t^{2}}=\boldsymbol{f}(\boldsymbol{r})-J \frac{\mathrm{d} \boldsymbol{r}}{\mathrm{d} t}+\boldsymbol{\eta}(t)
$$

providing that individuals are sufficiently sensitive to behavior of their neighbors, where $\boldsymbol{f}(\boldsymbol{r})$ is the cohesive force; $J$ denotes the strength of alignment; and $\boldsymbol{\eta}$ is a random perturbation of the velocity with strength $\epsilon_{v}$ and a $\delta$-correlated time dependence.

\subsection{Self-consistent calculation of of cohesive force}

The attractive interaction force acting on one body due to a system of bodies is the neighbor-joining harmonic spring. One essential approximation is to replace the many-fish problem by the problem of solving the motion of one fish in a certain self-consistent field. The cohesive force is then written in the following form by the one-body approximation:

$$
\boldsymbol{f}(\boldsymbol{r})=-\frac{\tilde{k}}{N^{\alpha}} \boldsymbol{r},
$$

where $\tilde{k} N^{-\alpha}$ is the effective spring constant for the springs strung out from the center of the school to a fish: the number of consecutive springs joining the fish to the center of the school via other companions is proportional to $N^{\alpha}$. The total "elastic energy" of inter-fish bonds in the school depends linearly on number $N$ of individuals in the school. The overall elastic energy after integration over a sphere of radius $R$ (denoting the average radius of the school) results $E_{\text {el }} \propto R^{4} N^{-\alpha}$, which is derived from the ideal harmonic spring, Eq.(25). Therefore, the exponent $\alpha$ will be determined self-consistently,

$$
\frac{R^{4}}{N^{\alpha}} \propto N
$$

The solution of the Langevin equation (24) with Eq.(25) takes the following asymptotic forms (Hori, 1977):

$$
\begin{gathered}
\sigma_{v}^{2}\left[\equiv\left\langle(\mathrm{d} \boldsymbol{r} / \mathrm{d} t)^{2}\right\rangle\right] \approx 3 \epsilon_{v} / J \\
\left\langle\boldsymbol{r}^{2}\right\rangle \approx \frac{\sigma_{v}^{2}}{\tilde{k}} N^{\alpha}
\end{gathered}
$$


where the root-mean-square $\sqrt{\left\langle\boldsymbol{r}^{2}\right\rangle}$ gives the average radius of the school, $R$. From Eq.(26) together with Eq.(28), the self-consistent value of the exponent $\alpha$ is obtained:

$$
\alpha=1 \text {. }
$$

As a consequence, the self-consistent cohesive force reads

$$
\boldsymbol{f}(\boldsymbol{r})=-\frac{\sigma_{v}^{2}}{b^{2} N} \boldsymbol{r}
$$

where $b$ denotes the effective inter-fish distance (a constant independent of $N): b^{2} \equiv \sigma_{v}^{2} / \tilde{k}$. The average radius of the school is then given by $b N^{1 / 2}$. Note that Eq.(27) is an example of a more general principle called the fluctuationdissipation theorem (Kubo, 1966).

Notice that the above model has been developed in the absence of the excluded volume interactions. Social cohesion by harmonic spring interactions between neighboring fish reminds us of the classical picture of a polymer chain based on the bead-spring model (Rouse, 1953). The exponent $\nu$ close to the value $3 / 5$ may then be understood by taking the excluded volume effect (Doi and Edwards, 1986). Such a school without excluded volume effect may be called the "Gaussian" school in line with polymer physics.

\subsection{The excluded volume effect}

From Eq.(24), the probability of the school radius being between $r$ and $r+\mathrm{d} r$ is given by the following (Hori, 1977):

$$
\Psi_{0}(r)=4 \pi r^{2}\left(\frac{3}{2 \pi b^{2} N}\right) \exp \left(-\frac{3 r^{2}}{2 b^{2} N}\right)
$$

in a stationary state in three dimensions (i.e. the position vector $\boldsymbol{r}$ follows a Gaussian distribution). The Gaussian school model considered above permits fish to occupy the same region in space. Of course this is a physical impossibility since each fish possesses its own finite volume. Therefore, in the "excluded volume" school, there are a number of Gaussian school configurations which are disallowed due to the steric effect. Let $p(r)$ be the probability that a Gaussian school configuration, as counted in Eq.(31), is also allowable under the excluded volume condition. We now calculate the probability that no overlaps occur when we place $N$ fish within a region of volume $\left(\sim r^{3}\right)$, which will lead to an estimation for $p(r)$. The approach is due to Doi (1996). Letting $w$ be the volume which is effectively excluded to one fish by the presence of another $\left(w \lesssim b^{3}\right)$, the probability that one particular fish will not overlap with another is then given by $\left(1-w / r^{3}\right)$. Since there are $N(N-1) / 2$ possible combinations 
of pairs, the probability that no overlap occurs in all of these combinations is given by

$$
p(r)=\left(1-w / r^{3}\right)^{N(N-1) / 2}=\exp \left(-\frac{w N^{2}}{2 r^{3}}\right),
$$

where $r^{3} \gg w$ and $N \gg 1$. Therefore, the probability distribution of the school radius $r$ can then be written as

$$
\Psi(r)=\Psi_{0}(r) p(r) \propto r^{2} \exp \left(-\frac{3 r^{2}}{2 b^{2} N}-\frac{w N^{2}}{2 r^{3}}\right)
$$

for the excluded volume school.

Both $\Psi_{0}(r)$ and $\Psi(r)$ have a maximum at certain values of $r$. Let us estimate the average size of the school radius in each model by calculating the positions of these maxima. The maximum of $\Psi_{0}(r)$ occurs at $R_{0}=\left(2 b^{2} N / 3\right)^{1 / 2}$. The maximum of $\Psi(r)$ occurs at $R$, which satisfies the following equation obtained by differentiating the logarithm of Eq.(33):

$$
\left(\frac{R}{R_{0}}\right)^{5}-\left(\frac{R}{R_{0}}\right)^{3}=\frac{9 \sqrt{6}}{16} \frac{w \sqrt{N}}{b^{3}} .
$$

If $N \gg 1$, the second term on the left-hand side of Eq.(34) can be neglected and hence

$$
R \simeq R_{0}\left(\frac{w \sqrt{N}}{b^{3}}\right)^{1 / 5} \propto N^{3 / 5} .
$$

The exponent $3 / 5$ is the very value extracted through the data collapse.

The scaling in the relationship between geometric dimensions and biomass of pelagic fish schools is analogous to that developed in polymer physics (de Gennes, 1979). The result suggests that the dynamics are unexpectedly "simple" and depend primarily on common cohesive motion in these animate and inanimate systems. Though the above is a very rough theory of school conformation with the excluded volume effect, the overall statistical properties do not depend on the details of the model, which is a consequence of universality (Stanlev, 1995).

\subsection{Exploiting the analogy to a polymer chain model}

As indicated in the Introduction, large internal variations in packing density occur within a school. Fréon and Misund (1999) pointed out that a source of substantial variation in internal school structure is the formation of subgroups. Such subgroups have been observed in saithe schools (Partridge, 1981), and in schools of minnow Phoxinus phoxinus (Pitcher, 1973) and herring 
(Pitcher and Partridge, 1979). Relatively independent movements of such clusters of individuals can open up empty spaces and cause large variation in school volume.

The polymer-chain analogue of the subgroup in school conformation is the "blob" (de Gennes, 1979): the polymer chain behaves as a series of blobs. Based on the blob concept, for small value of the excluded volume $w$, a subgroup within a school, with a number of $\lambda_{\mathrm{B}}$ of fish, must be nearly Gaussian. We see this from Eq.(33) when we find no effect of $w$ if $w \lambda_{\mathrm{B}}^{1 / 2} / b^{3}<1$. There is a certain value of $\lambda_{\mathrm{B}}\left[\sim\left(b^{3} / w\right)^{2}\right]$, beyond which excluded volume effects become important. A single school will appear Gaussian at scales $r<r_{\mathrm{B}}$ where

$$
r_{\mathrm{B}} \simeq b \lambda_{\mathrm{B}}^{1 / 2} \simeq b^{4} / w,
$$

while at scales $r>r_{\mathrm{B}}$ it will show excluded volume effects. According to the "blob" approach, an $N$-sized school can be described as a cluster of $N / \lambda_{\mathrm{B}}$ subgroups. Inside the subgroup the core repulsion (by excluded volume effects) is a weak perturbation leading to a Gaussian state. The school radius is then written as $R \simeq r_{\mathrm{B}}\left(\lambda_{\mathrm{B}}^{-1} N\right)^{\nu}$ in the native state.

Notice the plasticity of school geometry and internal structure. The compact, dense packing, as observed in artificial environments like small tanks, may be caused by the very strong stress. When the school is confronted by danger such as predators, the inter-fish distance decreases rapidly and all the vacuoles within the school collapse quickly (Fréon et al., 1992). This change may be closely related to the coil-globule transition in a polymer solution (Ptitsvn et al., 1968; de Gennes, 1975).

In real schools the nature of the short range interaction is quite complicated like van der Waals-type inter-molecular forces. Accordingly, all fish within a school interact via the two-body potential deformed from a parabolic potential of harmonic spring [grouping forces have been expressed as the gradient of a potential function in previous model studies (Mogilner et al., 2003)]. The potential change, denoted by $\Delta U_{i j}$, is given as the correction term to cohesive interaction potential between the $i$-th and the $j$-th fish: $\Delta U_{i j}$ will include steric effects and also may involve weak attractions. The correction factor to the probability distribution $\Psi(r)$ of the school radius $r$, in place of Eq.(32), is then given by the configuration integral

$$
p(r) \simeq r^{-3 N} \int \cdots \int \exp \left(-J \sum_{i<j} \Delta U_{i j} / \epsilon_{v}\right) \mathrm{d} \boldsymbol{x}_{1} \cdots \mathrm{d} \boldsymbol{x}_{N},
$$

where $\boldsymbol{x}_{i}$ denotes the position vector of the $i$-th fish, and $\exp \left(-J \Delta U_{i j} / \epsilon_{v}\right)$ is integrated over the school configuration space. We can estimate $p(r)$ by means of the cluster expansion (Maver and Maver, 1940; Uhlenbeck and Ford, 1962). 
Introducing a function of separation between pairs, $\boldsymbol{r}_{i j}=\boldsymbol{x}_{j}-\boldsymbol{x}_{i}$,

$$
\zeta_{i j}=\exp \left(-J \Delta U_{i j} / \epsilon_{v}\right)-1
$$

we integrate Eq.(37) in the Mayer cluster expansion over diagrams up to the second irreducible cluster, yielding

$$
p(r) \simeq \exp \left(-\frac{w N^{2}}{2 r^{3}}-\frac{w_{\Delta} N^{3}}{6 r^{6}}\right)
$$

with the excluded volume parameter

$$
w=-\int \zeta_{12} \mathrm{~d} \boldsymbol{r}_{12}
$$

and the other parameter defined by

$$
w_{\Delta}=-\iint \zeta_{12} \zeta_{13} \zeta_{23} \mathrm{~d} \boldsymbol{r}_{12} \mathrm{~d} \boldsymbol{r}_{13}
$$

The actual configuration is decided by maximizing the distribution function $\Psi(r)=\Psi_{0}(r) p(r)$, and this maximization condition leads to the equation determining the school radius $R$,

$$
\left(\frac{R}{R_{0}}\right)^{5}-\left(\frac{R}{R_{0}}\right)^{3}-\frac{27 w_{\Delta}}{16 b^{6}}\left(\frac{R}{R_{0}}\right)^{-3}=\frac{9 \sqrt{6}}{16} \frac{w \sqrt{N}}{b^{3}}
$$

Notice that, when the first irreducible cluster integral in Mayer's expansion is only taken into account, the configuration integral, Eq.(37), is evaluated by Eq.(32). In case that $\Delta U_{i j}$ consists of a hard core repulsion and a shortrange weak attraction (i.e. at the distance of near-collision $\left(r \sim w^{1 / 3}\right)$ the attraction gradient between fish becomes steeper), Eq.(40) is estimated as $w=w_{0}\left(1-\epsilon_{\Theta} / \epsilon_{v}\right)$, expressing the dependence of $w$ on the velocity-fluctuation $\epsilon_{v}$ of fish, where $w_{0}$ and $\epsilon_{\Theta}$ are constants (Doi and Edwards, 1986). During predation threats or laboratory observations, the value of excluded volume parameter $w$ will change sign from positive to negative, provided that the velocity fluctuation $\epsilon_{v}$ is suppressed below a certain value $\epsilon_{\Theta}$. Decreasing $\epsilon_{v}$ below $\epsilon_{\Theta}$, the dimensional size of the school becomes much smaller than that of a Gaussian school, as depicted in Fig.4. According to Eq.(42) the so called "expansion factor" $R / R_{0}$ is determined not by $w$ but by $w N^{1 / 2}$, and so if $N$ is large only a small change in $\epsilon_{v}$ will cause a big change in dimensional size. For example, for school of $10^{6}$ fish, a small variation in $\epsilon_{v}$ will induce a dramatic change in the school radius. Equation (42) states that when $-w N^{1 / 2} / b^{3} \gg 1$, $R / R_{0}$ is proportional to $\left(-w N^{1 / 2}\right)^{-1 / 3}$ and hence the solution is as follows:

$$
R \simeq R_{0}\left(\frac{-w b^{3} \sqrt{N}}{w_{\Delta}}\right)^{-1 / 3} \propto N^{1 / 3}
$$




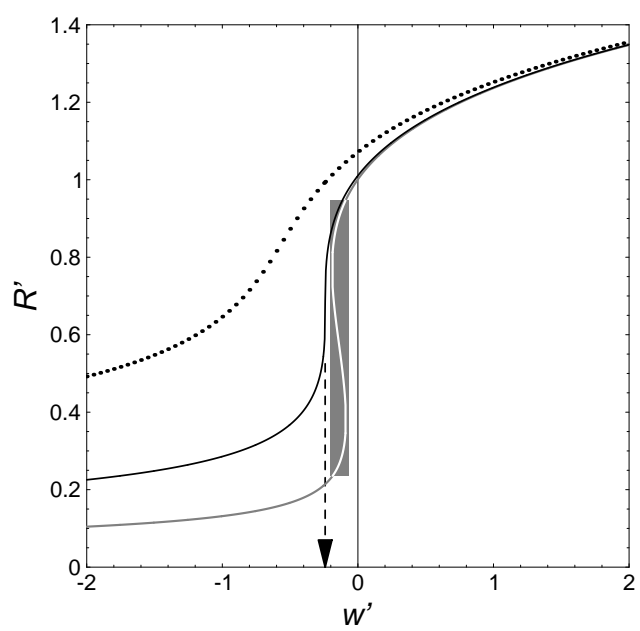

Fig. 4. Swollen-dense packing transition in the school geometry [the solution of Eq.(42)]. Here $R^{\prime}=R / R_{0}$ is plotted as a function of a combined variable $w^{\prime}=(9 \sqrt{6} / 16) w N^{1 / 2} / b^{3}$. Shown is the transition region $|w| N^{1 / 2} / b^{3} \approx 1$. At $\epsilon_{v}=\epsilon_{\Theta}$ the excluded volume parameter $w$ equals 0 . The repulsive excluded volume effect then balances the attractive forces between fish, and the school behaves as a Gaussian school. The property of the swollen-dense packing transition is quite different in the two regions separated by the critical value $w_{\Delta} / b^{6}=0.0228$. The transition between the the swollen conformation and the compact packing is gradual when $w_{\Delta} / b^{6}>0.0228$ (dotted line), while it becomes extremely sharp and the transition is discontinuous when $w_{\Delta} / b^{6}<0.0228$ (gray line). The solid line shows the marginal transition behavior, and the crossover takes place when $w^{\prime}=-0.242$ (indicated by the arrow). Notice that the solution of Eq.(42) is multivalued [depicted by a sigmoid curve (white cutout)], showing a first-order discrete phase change (Ptitsvn et al., 1968; de Gennes, 1975), when $w_{\Delta} / b^{6}<0.0228$.

which shows the closest packing of fish in a school, i.e. the constant density independent of the number of individuals. This change may be called the swollen-dense packing transition of schools.

\subsection{Configuration inside a swollen school}

The approach of treating fish schools as interacting particle systems naturally leads to the idea of applying successful methods of statistical physics to the description of moving together without a leader (Vicsek, 2001). In statistical physics, the presence of non-trivial scaling is usually taken to mean that the dynamics are largely governed by simple geometric properties of the system and do not depend strongly on detailed properties of the system components (Wilson, 1983).

As for the school geometry, the basic units are the effective inter-fish length $b$ and the number of fish, $N$. We assemble $\lambda$ neighboring fish in a school into 
$N / \lambda$ groups, e.g. in the sense of the Voronoi-Dirichlet diagram (Dirichlet, 1850; Voronoi, 1908, 1909). The length between centers of neighboring subgroups is defined by $\lambda^{\tilde{\nu}} b$ with an exponent $\tilde{\nu}$. After such a scale change as

$$
N \rightarrow \lambda^{-1} N, \quad b \rightarrow \lambda^{\tilde{\nu}} b,
$$

the macroscopic quantities which determine the overall properties of the school satisfy

$$
g\left(\lambda^{-1} N, \lambda^{\tilde{\nu}} b\right)=\lambda^{\chi} g(N, b),
$$

where $\chi$ is an exponent which depends on the quantity under consideration. For this to hold true for arbitrary $\lambda$, the function $g(N, b)$ must take the scaling form

$$
g(N, b)=N^{-\chi} g^{(\mathrm{sc})}\left(b N^{\tilde{\nu}}\right)
$$

where a new function $g^{(\mathrm{sc})}$ is introduced. This is the Widom-Kadanoff scaling law in critical phenomena (Goldenfeld, 1992). For example, the average radius of the school, $R$, should be unaltered under the transformation of Eq.(44):

$$
R(N, b)=R\left(\lambda^{-1} N, \lambda^{\tilde{\nu}} b\right)=R^{(\mathrm{sc})}\left(b N^{\tilde{\nu}}\right) .
$$

Because of Eq.(5), $R^{(\mathrm{sc})}(x)=x$ and we must have

$$
\tilde{\nu}=\nu
$$

where $\nu=3 / 5$ in three dimensions.

As an example of the application of the above scaling law, let us consider the pair correlation function inside a school, where fish movement take place in a three-dimensional space. A pair correlation function $\rho_{2}(r)$ is defined as follows. We pick one fish at random in the school, and we place it at origin. Then we ask, what is the number density of other fish at a (directional) distance $\boldsymbol{r}$ from the first, and we average the result over all choices of the first fish (and of directions). The function $\rho_{2}(r)$ has an integral which is just the total number of fish per school, $\int \rho_{2}(r) \mathrm{d} \boldsymbol{r}=N$. Hence, from dimensional analysis, we can write

$$
\rho_{2}(r)=R^{-3} g(N, r / b) .
$$

Under the transformation that neighboring $\lambda$ fish are grouped to form one subunit in a school, $\rho_{2}(r)$ will be reduced by $1 / \lambda$, since the pair correlation function is proportional to the number density of fish. Therefore

$$
g\left(\lambda^{-1} N, r / \lambda^{\nu} b\right)=\lambda^{-1} g(N, r / b) .
$$

Then, the function $\rho_{2}(r)$ obeys a simple scaling rule

$$
\rho_{2}(r)=N R^{-3} \rho_{2}^{(\mathrm{sc})}(r / R),
$$


where $\rho_{2}^{(\mathrm{sc})}$ is a dimensionless universal function, and $N R^{-3}$ is the dimensional factor. Focusing on the limit $r \ll R$, we can reach the form of $\rho_{2}(r)$ by a simple argument. In a sphere of radius $r$ we have a certain number of fish, $\tilde{N}$, related to $r$ by the excluded volume exponent: $\tilde{N}^{3 / 5} b \simeq r$. The function $\rho_{2}(r)$ scales like the density of fish in the sphere,

$$
\rho_{2}(r) \simeq \frac{\tilde{N}}{r^{3}} \simeq \frac{1}{r^{4 / 3} b^{5 / 3}} \quad(\text { for } r<R),
$$

which gives $\rho_{2}^{(\mathrm{sc})}(x) \simeq x^{-4 / 3}$.

The possible observable property, Eq.(51), tells us that if we were to measure $\rho_{2}(r)$ for fish schools of different size $N$, there would be superposition of the curves obtained by plotting $\rho_{2}(r) R^{3} / N$ against $r / R$. Thus this kind of scaling relation may be verified experimentally on large schools of pelagic fish.

\section{Discussion}

The power-law scaling generally exists in dimension-to-biomass relationship of pelagic fish schools in nature. Here I have estimated the power-law exponent $\nu$ of the geometric relation, based on the dependence of the power-law regime of the school-dimension distribution $W^{(\mathrm{G})}(R)$ on $\nu$, i.e. Eq.(11). We have tested whether the distributions $W^{(\mathrm{G})}(R)$ are self-similar (i.e. exhibit scaling), relying on both the power law for the dimension-to-biomass relationship of Eq.(5) and the FSS relation in the power-law school-biomass distribution of Eq.(6). Plotting the scaled histogram-data from the 22 in-situ observations, we have found that the 22 curves do indeed collapse onto each other (Fig.2a), suggesting that $W^{(\mathrm{G})}(R)$ follows a universal functional form [Eq.(8)]. We have extracted the power-law exponent of dimension-to-biomass scaling relationship via a minimization of a measure to quantify the nature of scaling collapse. Next I have explained the value of the exponent $\nu$, proposing the Gaussian school model for the fish with excluded volume $w$. By exploiting the analogy between fish-school and polymer conformations, we have examined the behavioral algorithm governing the swollen conformation of large-sized schools. We have seen that the exponent is modified strongly by the steric effect. What is universal in Eq.(5) is the exponent $\nu \approx 0.6$ : it is independent of species as well as environmental conditions, and the same for all schools. The constant that multiplies $N^{\nu}$ in Eq.(5) is non-universal $\left[\sim\left(w b^{2}\right)^{1 / 5}\right.$ with effective inter-fish distance $b$, predicted from Eq.(35)], and depends on the details of interactions between fish which may vary with species and environmental conditions (Morgan, 1988).

To understand the scaling law for school geometry, Eq.(5) with $\nu=3 / 5$, it is 
essential to see what the value of $\nu$ represents. Obviously, for a regular object embedded into a $d$-dimensional Euclidean space, Eq.(5) would have the form $N(R) \sim R^{d}$ expressing the fact that the volume of a $d$-dimensional object grows with its linear size $R$ as $R^{d}$. Contrary to an integer dimensionality, it has been shown that the packing structure within the schools is characterized by a non-integer (i.e. fractal) dimensionality of $1 / \nu \approx 1.7$ : the number of fish in a school of radius $R$ scales as $N(R) \sim R^{1 / \nu}$. During the last decades of the twentieth century it has widely been recognized by researchers working in diverse areas of science that many of the structures commonly observed possess a rather special kind of geometrical complexity; the name "fractal" was coined by Benoit Mandelbrot for these complex shapes. Objects of biological origin are many times fractal-like (Vicsek (Ed.), 2001).

FSS plots of data lead to possibly important and practical insights in ecology. In the context of the fisheries mission, the demonstrated geometric relation between dimensions and biomass of pelagic fish schools, when applied to mapping pelagic schooling fish, will largely improve the precision in the fish stock assessment. Because of the linear dependence of the fish population on $\langle N\rangle_{P}$, as given by Eq.(23), the fish stock abundance can be inferred from an index (i.e. cut-off size) that can be determined directly from observations [see Eq.(7)].

I am very grateful to Leah Edelstein-Keshet for helpful suggestions.

\section{References}

Aji, V., Goldenfeld, N., 2001. Fluctuations in finite critical and turbulent systems. Phys. Rev. Lett. 86, 1007-1010., doi:10.1103/PhysRevLett.86.1007.

Anderson, J. J., 1981. A stochastic model for the size of fish schools. U.S. Fish. Bull. 79, 315-323.

Aoki, I., 1980. An analysis of the schooling behavior of fish: internal organization and communication process. Bull. Ocean Res. Inst. Univ. Tokyo 12, $1-65$.

Aoki, I., Inagaki, T., 1988. Photographic observations on the behavior of Japanese anchovy Engraulis japonica at night in the sea. Mar. Ecol. Prog. Ser. 43, 213-221.

Azovsky, A. I., 2000. Concept of scale in marine ecology: linking the words or the worlds? Web Ecol. 1, 28-34.

Banavar, J. R., Green, J. L., Harte, J., Maritan, A., 1999. Finite size scaling in ecology. Phys. Rev. Lett. 83, 4212-4214.

Beecham, J. A., Farnsworth, K. D., 1999. Animal group forces resulting from predator avoidance and competition minimization. J. Theor. Biol. 198, 533548. 
Binder, K., Heermann, D. W., 1988. Monte Carlo Simulation in Statistical Physics. Springer-Verlag, Berlin.

Bonabeau, E., Dagorn, L., 1995. Possible universality in the size distribution of fish schools. Phys. Rev. E 51, R5220-R5223.

Bonabeau, E., Dagorn, L., Fréon, P., 1998. Space dimension and scaling in fish school-size distributions. J. Phys. A: Math. Gen. 31, L731-L736.

Bonabeau, E., Dagorn, L., Fréon, P., 1999. Scaling in animal group-size distribution. Proc. Natl. Acad. Sci. USA 96, 4472-4477.

Breder, Jr., C. M., 1954. Equation descriptive of fish schools and other animal aggregations. Ecology 35, 361-370.

Camacho, J., Guimerà, R., Amaral, L. A. N., 2002. Robust patterns in food web structure. Phys. Rev. Lett. 88, 228102., doi:10.1103/PhysRevLett.88.228102.

Camazine, S., Deneubourg, J.-L., Franks, N. R., Sneyd, J., Theraulaz, G., Bonabeau, E., 2001. Self-Organization in Biological Systems. Princeton University Press, Princeton.

Chave, J., Levin, S., 2003. Scale and scaling in ecological and economic systems. Environ. Resource Econ. 26, 527-557.

Coetzee, J., 2000. Use of a shoal analysis and patch estimation system (SHAPES) to characterise sardine schools. Aquat. Living Resour. 13, 1-10.

Cushing, D. H., 1977. Observations of fish schools with the ARL scanner. Rapp. P.-v Réun. Cons. int. Explor. Mer 170, 15-20.

de Gennes, P.-G., 1975. Collapse of a polymer chain in poor solvents. J. de Physique 36, L55-L57.

de Gennes, P.-G., 1979. Scaling Concepts in Polymer Physics. Cornell University Press, Ithaca, New York.

Dill, L. M., Dunbrack, R. L., Major, P. F., 1981. A new stereophotographic technique for analyzing the three-dimensional structure of fish schools. Env. Biol. Fish. 6, 7-13.

Dirichlet, G. L., 1850. Über die Reduktion der positiven quadratischen Formen mit drei unbestimmten ganzen Zahlen. J. Reine Angew. Math. 40, 209-227.

Doi, M., 1996. Introduction to Polymer Physics. Oxford University Press, New York.

Doi, M., Edwards, S. F., 1986. The Theory of Polymer Dynamics. Oxford University Press, New York.

Fisher, M. E., Barber, M. N., 1972. Scaling theory for finite-size effects in the critical region. Phys. Rev. Lett. 28, 1516-1519.

Flory, P., 1953. Principles of Polymer Chemistry. Cornell University Press, Ithaca, New York.

Fréon, P., Gerlotto, F., Soria, M., 1992. Changes in school structure according to external stimuli: description and influence on acoustic assessment. Fish. Res. 15, 45-66.

Fréon, P., Misund, O. A., 1999. Dynamics of Pelagic Fish Distribution and Behaviour: Effects on Fisheries and Stock Assessment. Fishing News Books, Blackwell, Osney Mead, Oxford. 
Goldenfeld, N., 1992. Lectures on Phase Transitions and the Renormalization Group. Vol.85 of Frontiers in Physics. Addison-Wesley, Reading, Massachusetts.

Graves, J., 1976. Photographic method for measuring spacing and density within pelagic fish schools at sea. U.S. Fish. Bull. 75, 230-234.

Hamburger, D. A., Biham, O., Avnir, D., 1996. Apparent fractality emerging from models of random distributions. Phys. Rev. E 53, 3342-3358.

Halley, J. M., Hartley, S., Kallimanis, A. S., Kunin, W. E., Lennon, J. J., Sgardelis, S. P., 2004. Uses and abuses of fractal methodology in ecology. Ecol. Lett. 7, 254-271., doi:10.1111/j.1461-0248.2004.00568.x.

Hara, I., 1984. Distribution and school size of Japanese sardine in the waters off the southeastern coast of Hokkaido on the basis of echo sounder surveys. Bull. Tokai Reg. Fish. Res. Lab. 113, 67-78.

Hara, I., 1986. Stock assessment of Japanese sardine in the waters off the southeast coast of Hokkaido using line transect method. Bull. Jpn. Soc. Sci. Fish. 52, 69-73.

Hara, I., 1990. Stock Assessment and Migration of Japanese Sardine Schools in the Waters off the Southeastern Coast of Hokkaido. Ph.D. Thesis, Kyoto University, Kyoto, Japan.

Harte, J., Kinzig, A., Green, J., 1999. Self-similarity in the distribution and abundance of species. Science 284, 334-336.

Higashi, M., Yamamura, N., 1993. What determines animal group size? insider-outsider conflict and its resolution. Am. Nat. 142, 553-563.

Hoare, D. J., Couzin, I. D., Godin, J.-G. J., Krause, J., 2004. Contextdependent group size choice in fish. Anim. Behav. 67, 155-164., doi:10.1016/j.anbehav.2003.04.004.

Hori, J., 1977. Langevin Equation. Iwanami Shoten, Publ., Tokyo.

Keitt, T. H., Stanley, H. E., 1998. Dynamics of North American breeding bird populations. Nature 393, 257-260.

Keitt, T. H., Amaral, L. A. N., Buldyrev, S. V., Stanley, H. E., 2002. Scaling in the growth of geographically subdivided populations: invariant patterns from a continent-wide biological survey. Phil. Trans. R. Soc. Lond. B 357, 627-633., doi:10.1098/rstb.2001.1013.

Kubo, R., 1966. The fluctuation-dissipation theorem. Rep. Prog. Phys. 29 (1), 255-284.

Lillo, F., Farmer, J. D., Mantegna, R. N., 2002. Single curve collapse of the price impact function for the New York Stock Exchange. preprint cond-mat/0207428 at 〈http://xxx.lanl.gov

Lillo, F., Farmer, J. D., Mantegna, R. N., 2003. Master curve for price-impact function. Nature 421, 129-130.

MacArthur, R. H., Wilson, E. O., 1967. The Theory of Island Biogeography. Princeton University Press, Princeton.

Mandelbrot, B. B., 1982. The Fractal Geometry of Nature. W. H. Freeman and Co., New York.

May, R. M., 1999. Unanswered questions in ecology. Phil. Trans. R. Soc. Lond. 
B 354, 1951-1959.

Mayer, M. G., Mayer, J. E., 1940. Statistical Mechanics. John Wiley \& Sons, New York.

Misund, O. A., 1990. Sonar observations of schooling herring: school dimensions, swimming behaviour, and avoidance of vessel and purse seine. Rapp. P.-v Réun. Cons. int. Explor. Mer 189, 135-146.

Misund, O. A., 1993a. Dynamics of moving masses: variability in packing density shape and size among herring sprat and saithe schools. ICES J. Mar. Sci. 50, 145-160.

Misund, O. A., 1993b. Abundance estimation of fish schools based on a relationship between school area and school biomass. Aquat. Living Resour. 6, 235-241.

Misund, O. A., Aglen, A., Beltestad, A. K., Dalen, J., 1992. Relationship between the geometric dimensions and biomass of schools. ICES J. Mar. Sci. 49, 305-315.

Misund, O. A., Aglen, A., Frønæs, E., 1995. Mapping the shape, size, and density of fish schools by echo integration and a high-resolution sonar. ICES J. Mar. Sci. 52, 11-20.

Misund, O. A., Aglen, A., Hamre, J., Ona, E., Røttingen, I., Skagen, D., Valdemarsen, J. W., 1996. Improved mapping of schooling fish near the surface: comparison of abundance estimates obtained by sonar and echo integration. ICES J. Mar. Sci. 53, 383-388.

Misund, O. A., Coetzee, J., 2000. Recording fish schools by multi-beam sonar: potential for validating and supplementing echo integration recordings of schooling fish. Fish. Res. 47, 149-159., doi:10.1016/S0165-7836(00)00166-1.

Misund, O. A., Coetzee, J., Fréon, P., Gardener, M., Olsen, K., Svellingen, I., Hampton, I., 2003. Schooling behaviour of sardine Sardinops sagax in False Bay, South Africa. Afr. J. Mar. Sci. 25, 185-193.

Misund, O. A., Floen, S., 1993. Packing density structure of herring schools. ICES Mar. Sci. Symp. 196, 26-29.

Mogilner, A., Edelstein-Keshet, L., Bent, L., Spiros, A., 2003. Mutual interactions, potentials, and individual distance in a social aggregation. J. Math. Biol. 47, 353-389., doi:10.1007/s00285-003-0209-7.

Morgan, M. J., 1988. The effect of hunger, shoal size and the presence of a predator on shoal cohesiveness in bluntnose minnows, Pimephales notatus Rafinesque. J. Fish. Biol. 32, 963-971.

Niwa, H.-S., 1994. Self-organizing dynamic model of fish schooling. J. Theor. Biol. 171, 123-136.

Niwa, H.-S., 1996a. Newtonian dynamical approach to fish schooling. J. Theor. Biol. 181, 47-63.

Niwa, H.-S., 1996b. Mathematical model for the size distribution of fish schools. Computers Math. Applic. 32 (11), 79-88.

Niwa, H.-S., 1998. School size statistics of fish. J. Theor. Biol. 195, 351-361.

Niwa, H.-S., 2003. Power-law versus exponential distributions of animal group sizes. J. Theor. Biol. 224, 451-457., doi:10.1016/S0022-5193(03)00192-9. 
Niwa, H.-S., 2004a. Space-irrelevant scaling law for fish school sizes. J. Theor. Biol. 228, 347-357., doi:10.1016/j.jtbi.2004.01.011.

Niwa, H.-S., 2004b. Exploitation dynamics of fish stocks. Meeting Abstracts of the Physical Society of Japan 59 (2), Part 2, p.201. paper submitted to Ecol. Econ.

Okubo, A., Chiang, H. C., 1974. An analysis of the kinematics of swarming of Anarete pritchardi Kim (Diptera: Cecidomyiidae). Res. Popul. Ecol. 16, $1-42$.

Okubo, A., Levin, S. A., 2001. Diffusion and Ecological Problem: Modern Perspectives. 2nd ed. Springer-Verlag, New York.

Parr, A. E., 1927. A contribution to the theoretical analysis of the schooling behavior of fishes. Bulletin of the Bingham Oceanographic Collection 1 (1), $1-32$.

Parrish, J. K., Hamner, W. M. (Eds.), 1997. Animal Groups in Three Dimensions. Cambridge University Press, Cambridge.

Partridge, B. L., 1980. The effect of school size or the structure and dynamics of minnow schools. Anim. Behav. 28, 68-77.

Partridge, B. L., 1981. Internal dynamics and interrelations of fish in schools. J. Comp. Physiol. 144, 313-325.

Partridge, B. L., 1982. The structure and function of fish schools. Sci. Am. 246, 90-99.

Pitcher, T. J., 1973. The three-dimensional structure of fish schools in the minnow, Phcxinus phcxinus. Anim. Behav. 21, 673-686.

Pitcher, T. J., Parrish, J. K., 1993. Functions of shoaling behaviour in teleosts, in: Pitcher, T. J. (Ed.), Behaviour of Teleost Fishes. 2nd ed. Chapman \& Hall, London, Ch.12, pp.363-439.

Pitcher, T. J., Partridge, B. L., 1979. Fish school density and volume. Mar. Biol. 54, 383-394.

Preston, F. W., 1962. The canonical distribution of commonness and rarity: Part I, and Part II. Ecology 43, 185-215, and 410-432.

Ptitsyn, O. B., Kron, A. K., Eizner, Y. Y., 1968. The models of the denaturation of globular proteins. I. theory of globula-coil transitions in macromolecules. J. Polym. Sci. C 16, 3509-3517.

Pulliam, H. R., Caraco, T., 1984. Living in groups: is there an optimal group size?, in: Krebs, J.R., Davies, N.B. (Eds.), Behavioural Ecology. An Evolutionary Approach. 2nd ed. Blackwell Science, Oxford, pp. 122-147.

Rinaldo, A., Maritan, A., Cavender-Bares, K. K., Chisholm, S. W., 2002. Cross-scale ecological dynamics and microbial size spectra in marine ecosystems. Proc. R. Soc. Lond. B 269, 2051-2059., doi: 10.1098/rspb.2002.2102.

Rouse, P. E., 1953. A theory of the linear viscoelastic properties of dilute solutions of coiling polymers. J. Chem. Phys. 21, 1272-1280.

Stanley, H. E., 1995. Power laws and universality. Nature 378, 554.

Symons, P. E. K., 1971. Spacing and density in schooling threespine sticklebacks (Gasterosteus aculeatus) and mummichog (Fundulus heteroclitus). J. Fish. Res. Bd. Can. 28, 999-1004. 
Tauti, M., Miyosi, K., 1929. On the movement of a fish-group. I. on the direction of a fish-group on passing the end of a wall along which it has moved. J. Imperial Fish. Inst. Jpn. 24, 73-76.

Tauti, M., Hudino, T., 1929. On the movement of a fish-group. II. the case when fishes were barred from their progress by a wall. J. Imperial Fish. Inst. Jpn. 24, 76-79.

Tauti, M., Yasuda, H., 1929. On the movement of a fish-group. III. the case when fishes were barred from their progress by a net. J. Imperial Fish. Inst. Jpn. 24, 80-86.

Tauti, M., Yasuda, H., 1930. On the movement of a fish-group. IV. the shape of the entrance of fishing net and the fish-group moving in and out. J. Imperial Fish. Inst. Jpn. 25, 41-50.

Tauti, M., Yasuda, H., 1933a. The direction in which a fish-group goes on after having met with a net. Bull. Jpn. Soc. Sci. Fish. 2, 17-19.

Tauti, M., Yasuda, H., 1933b. How will a fish-group be attracted? Bull. Jpn. Soc. Sci. Fish. 2, 124-127.

Uhlenbeck, G. E., Ford, G. W., 1962, The theory of linear graphs with applications to the theory of the virial development of the properties of gases, in: de Boer, J., Uhlenbeck, G. E. (Eds.), Studies in Statistical Mechanics. North-Holland, Amsterdam, Vol.1, Ch.2, pp.119-211.

Vicsek, T., 2001. A question of scale. Nature 411, 421.

Vicsek, T. (Ed.), 2001. Fluctuations and Scaling in Biology. Oxford University Press, Oxford.

Voronoi, G. M., 1908. Nouvelles applications des paramètres continus à la théorie des formes quadratiques deuxième mémoire: rechrches sur les parallélloèdres primitifs. J. Reine Angew. Math. 134, 198-287.

Voronoi, G. M., 1909. Deuxième mémoire: recherches sur les paralléloedres primitifs. J. Reine Angew. Math. 136, 67-181.

Warburton, K., Lazarus, J., 1991. Tendency-distance models of social cohesion in animal groups. J. Theor. Biol. 150, 473-488.

Wilson, K. G., 1983. The renormalization group and the critical phenomena. Rev. Mod. Phys. 55, 583-600. 